\title{
Control of ZnO nanowire arrays by Nanosphere Lithography (NSL) on laser-
} produced $\mathrm{ZnO}$ substrates.

S. Garry, E. McCarthy, J.-P. Mosnier, E. McGlynn*.

School of Physical Sciences and National Centre for Plasma Science and Technology

(NCPST),

\section{Dublin City University,}

Glasnevin, Dublin 9, Ireland.

\begin{abstract}
Nanosphere lithography (NSL) is a successful technique for fabricating highlyordered arrays of $\mathrm{ZnO}$ nanowires typically on sapphire and $\mathrm{GaN}$ substrates. In this work, we investigate the use of thin $\mathrm{ZnO}$ films deposited on $\mathrm{Si}$ by pulsed laser deposition (PLD) as the substrate. This has a number of advantages over the alternatives above, including cost and potential scalability of production and it removes any issue of inadvertent n-type doping of nanowires by diffusion from the substrate. We demonstrate ordered arrays of $\mathrm{ZnO}$ nanowires, on $\mathrm{ZnO}$-coated substrates by PLD, using a conventional NSL technique with gold as the catalyst. The nanowires were produced by Vapor Phase Transport (VPT) growth in a tube furnace system and grew only on the areas pre-patterned by $\mathrm{Au}$. We have also investigated the growth of $\mathrm{ZnO}$ nanowires using $\mathrm{ZnO}$ catalyst points deposited by $\mathrm{PLD}$ through an NSL mask on a bare silicon substrate.
\end{abstract}

*Author to whom correspondence should be addressed: enda.mcglynn@dcu.ie. 


\section{Introduction}

There is currently a great deal of interest in $1 \mathrm{D}$ nanoscale materials with the aim of developing nanodevices with improved functionality. One such potential application is in the development of new field emission (FE) sources for use as miniature $\mathrm{X}$-ray sources or flat panel displays [1,2]. Zinc oxide $(\mathrm{ZnO})$ is a promising candidate for such devices which has applications in various technologies [3] and in particular is well suited for FE applications. However, strong control of the morphology of nanowire arrays produced for this purpose is required. Array density has been shown to have a large impact on the efficiency and uniformity of FE devices [4]. This control over array morphology can be achieved using nanosphere lithography (NSL) and there are many examples in the literature of well ordered, periodical arrays of $\mathrm{ZnO}$ nanowires grown via the use of NSL [5-9] to create an initial ordered template of metal islands on a substrate. NSL relies on producing a lithographic mask comprised of a close packed monolayer of polystyrene beads which can be produced with differing aperture sizes and separation. It is often preferable to other patterning techniques such as e-beam lithography and mask lithography [9] due to its low cost and the ability to easily pattern large areas. This technique is most commonly used in conjunction with vapor phase transport growth for $\mathrm{ZnO}$ nanowires where, under suitable conditions, the nanowire growth occurs at the metal catalyst point only. NSL has been used to create well ordered arrays of $\mathrm{ZnO}$ nanowires on substrates such as sapphire [5-7] and GaN thin films [8], however to our knowledge this technique has not been applied to growth on $\mathrm{ZnO}$ substrates, $\mathrm{ZnO}$ buffer layers or even directly on Si substrates. The development of ordered arrays on these types of substrate would have a number of advantages such as removing the possibility of 
unwanted doping from the substrate (e.g., Al from sapphire substrates) and also allowing reductions in cost due to the use of a range of cheaper substrate materials and enable potential scalability to large area substrates [10]. While ordered growth on $\mathrm{ZnO}$ substrates is desirable, it presents problems which are not addressed in the literature since these same problems are not encountered for growth on substrates such as sapphire and GaN. This issue is that $\mathrm{ZnO}$ naturally presents a highly favourable surface for $\mathrm{ZnO}$ nucleation, causing growth to occur on all areas of the substrate making selective growth difficult or impossible. To overcome this issue, very close control over the Zn vapor pressure in the VPT growth is required.

In this paper we present the results of studies attempting to grow well ordered arrays of vertically aligned $\mathrm{ZnO}$ nanowires by VPT on $\mathrm{ZnO}$ buffer layers deposited by PLD onto $\mathrm{Si}(100)$ substrates. Ordered arrays were achieved by patterning the $\mathrm{ZnO}$ substrate with $\mathrm{Au}$ islands using NSL. These arrays cover large area substrates (of dimensions $>1 \mathrm{~cm} \times 1 \mathrm{~cm}$ ) and are uniform in terms of nanowire height and density of growth across these areas. The problem of growth over the entire substrate has been overcome by careful placement of the sample in the VPT tube furnace relative to the source materials. We also investigate the possibility of growth of patterned arrays of nanowires without the use of Au catalyst points onto Si substrates. This is a very desirable goal as it entirely removes the possibility of unwanted doping/contamination from the substrate or from metallic catalyst points.

\section{Experimental details}

Si (100) substrates were cleaned by ultrasonication in acetone and isopropanol for 15 minutes each and then a $\mathrm{ZnO}$ buffer layer was deposited by PLD. Due to the 
deposition of a $\mathrm{ZnO}$ buffer layer, the native oxide layer is not expected to impact result and no attempt was made to remove the native oxide prior to growth. The thickness of the $\mathrm{ZnO}$ buffer layer was maintained between $150 \mathrm{~nm}$ and $250 \mathrm{~nm}$. PLD was carried out with a frequency quadrupled Nd:YAG laser with a wavelength of 266 $\mathrm{nm}$ focussed on to a sintered compressed $\mathrm{ZnO}$ target. The fluence was kept at $\sim 3$ $\mathrm{J} / \mathrm{cm}^{2}$. The base pressure of the system was $\sim 5 \times 10^{-12} \mathrm{~Pa}\left(5 \times 10^{-8} \mathrm{mbar}\right)$ and depositions were carried out at $10 \mathrm{~Pa}(0.1 \mathrm{mbar})$ with a $30 \mathrm{sccm}$ flow of oxygen. The samples were heated to $900^{\circ} \mathrm{C}$ before deposition to remove any impurities and deposition was carried out at $300^{\circ} \mathrm{C}$. The temperature was then ramped up to $700^{\circ} \mathrm{C}$ to anneal the deposited layer and slowly cooled to room temperature.

These substrates were then coated with a close packed monolayer of polystyrene (PS) nanospheres. The spheres used (Thermo Scientific 5100A) were 1 $\mu \mathrm{m}$ in diameter. The nanosphere solution was mixed with ethanol in a 1:1 ratio and carefully applied to the surface of water in a Petri dish. Once in contact with the water surface the spheres are seen to disperse across the surface before organising into areas of close packed monolayer. The $\mathrm{ZnO}$ substrate is then immersed in the water and slowly withdrawn through an area where the spheres have packed together. The quality of this layer can be easily verified by visual inspection as these layers display very clear opalescence under white light illumination. The coated substrates are then allowed to dry in air before a layer of Au (of nominal thickness $2.5 \mathrm{~nm}$ ) is deposited over the nanosphere layer using a bell jar thermal evaporator. To control the size of the deposited $\mathrm{Au}$ islands the substrates were held at angles from 10 to 20 degrees relative to the Au source. This allows a rough degree of control over the size of the islands. The nanospheres are finally removed by ultrasonic bath in toluene, leaving only an ordered array of Au islands across the substrate. 
VPT growth was carried out on the Au patterned substrates using the method described in [11] at a temperature at $925^{\circ} \mathrm{C}$ for one hour and allowed to cool to room temperature over a period of several hours. $0.06 \mathrm{~g}$ of $\mathrm{ZnO}$ and $0.06 \mathrm{~g}$ graphite powder was mixed thoroughly using a pestle and mortar and loaded into an alumina boat with the substrate loaded facing down approximately $1 \mathrm{~cm}$ away from the source powders in the direction of the Ar flow (i.e. downstream).

For the growths carried out without the use of Au catalysts, cleaned Si (100) substrates were coated with a layer of close packed nanospheres as described above and then coated with a layer of $\mathrm{ZnO}$ by PLD. For these substrates, the deposition was carried out at room temperature as the polystyrene beads cannot withstand high temperatures. The spheres were removed by ultrasonic bath in toluene and VPT nanowire growth was carried out under the same conditions as above, but with the substrate loaded directly over the source powders as under these conditions growth on non-patterned bare $\mathrm{Si} / \mathrm{SiO}_{2}$ regions is not expected as $\mathrm{Si} / \mathrm{SiO}_{2}$ does not present an attractive surface for the nucleation of $\mathrm{ZnO}$.

Characterisation was carried out using scanning electron microscopy (KarlZeiss EVO series).

\section{Results and Discussion}

\subsection{Growth of ordered nanowire arrays on continuous PLD ZnO buffer layers using} NSL patterned Au catalysts.

Au was deposited through a NSL mask, leaving islands of Au patterned regions of 3 mm diameter which were then grown upon. Strong growth was seen on the samples 
once removed from the VPT furnace. Invariably there were three distinct regions after growth. The first region, that closest to the source powders, is white/grey in colour and is dominated by very strong nanowire growth, however this growth is unaligned and does not show any patterning. It is thought this is due to a high $\mathrm{Zn}$ vapour pressure in this region.

The second region is that where $\mathrm{ZnO}$ deposits solely on areas catalyzed with Au. Areas not patterned with $\mathrm{Au}$ are unchanged post-growth. Where catalyzed, the substrate appeared matt-white, an indicator of nanowire growth. The substrate also retained the opalescent qualities of the NSL mask used to pattern it. This is a strong indicator of spatially ordered growth, as deduced from comparison of SEM data with visual inspection. It is in this region where ordered, aligned nanowire growth occurs.

The final region is the furthest from the source powders. Some $\mathrm{ZnO}$ nucleation occurs here but does not develop into nanowires. Visually its appearance is unchanged. It is thought that this is due to low $\mathrm{Zn}$ vapour pressure, as it has been depleted through deposition on other regions of the substrate.

Growths were carried out using arrays of Au catalyst points deposited at various angles with respect to the $\mathrm{Au}$ source as described in [12]. This produced samples with $\mathrm{Au}$ islands of varying sizes depending on both the angle of deposition and the orientation of nanosphere domains on each sample. It was observed that on samples where $\mathrm{Au}$ was deposited perpendicular to the source that multiple nanowires would grow from each point in the catalyst pattern, in fact the pattern itself is obscured except for regions with quite short nanowires. Depositing $\mathrm{Au}$ at higher angles reduced multiwire nucleations but only to a certain degree. Au deposited at an angle of $\sim 10^{\circ}$ would result in nanowire arrays that would also have multiple 
nucleations from each point, however there were fewer nanowires growing from each point and the hexagonal pattern was still apparent. There are also regions where a single nanowire would grow from a single dot. Increasing the deposition angle to $\sim 16^{\circ}$ or higher further reduced the occurrence of multiple nucleation from each point, however it introduced another issue impacting the quality of the arrays grown. In this case areas of the sample were observed with no nanowire growth at all. It is suspected that this is due to the random orientation of nanosphere domains across the sample, causing some of the nucleation points to be too small to catalyze growth or possibly not be present at all.

Growths were also carried out with varying thicknesses of Au. Two nominal thicknesses were used, $2.5 \mathrm{~nm}$ and $5 \mathrm{~nm}$. The growths using the thicker, $5 \mathrm{~nm}$, coating were seen to be less spatially ordered than those coated with $2.5 \mathrm{~nm}$ thickness of Au. We believe that this is due to the thicker Au layers beading up at high temperature and moving slightly across the surface of the substrate, causing growth of multiple rods from each point and some growth from areas not initially patterned by Au.

The best results were achieved using $2.5 \mathrm{~nm}$ thick $\mathrm{Au}$ catalyst pattern deposited $\sim 10^{\circ}$ from normal as demonstrated in Fig 1 and Fig 2. These figures show areas of highly patterned nanowires in plan view with tilted views inset. It is clear from these images that there is multiple wire growth from many catalyst points and that there is no growth where the substrate is not catalysed with Au. Fig. 1 also includes XRD data showing $\theta-2 \theta$ and rocking curve of $\mathrm{ZnO}(0002)$ peak. In plan view the hexagonal pattern is clear and the majority of nanowires appear as points due to good alignment. There are also a number of defects in the pattern visible, including nanowires that are not perfectly aligned. 
The patterned arrays of nanowires however do not extend over the full substrate. The regions of successful growth are confined to regions that are approximately 4 or $5 \mathrm{~mm}$ wide and are found approximately $5 \mathrm{~mm}$ away from the source powders.

\subsection{Growth of nanowire arrays on NSL patterned ZnO buffer layers deposited by PLD} with no Au catalyst.

The samples were examined by SEM both before and after VPT growth. Once the nanosphere layer was removed from the sample it had the same appearance as a bare Si substrate. However when examined by SEM a $\mathrm{ZnO}$ honeycomb pattern was noted across the sample. Unlike Au deposition through a nanosphere mask, which results in discrete islands across the sample, depositing $\mathrm{ZnO}$ through this mask by PLD resulted in a honeycomb pattern. After VPT growth the sample had taken on a matt white appearance. SEM studies showed that randomly oriented $\mathrm{ZnO}$ nanowire growth occurred all over the sample and not solely at NSL patterned regions. Low temperature growth is needed as to not destroy the PS nanospheres, however it results in poor texture of $\mathrm{ZnO}$ seeds [13], which we expect caused unaligned growth. In areas near the edge of the substrate where nanowire growth did not occur it was clear that the $\mathrm{ZnO}$ honeycomb pattern formed a $\mathrm{ZnO}$ film when subjected to VPT growth. This film growth completely obscured the NSL pattern making patterned ordered arrays impossible to grow with this method. 


\section{Conclusions}

In summary, we have presented a procedure for producing well ordered arrays of vertically aligned $\mathrm{ZnO}$ nanowires on an NSL patterned $\mathrm{Au}$ catalyzed $\mathrm{ZnO}$ buffer layer deposited by PLD on Si. Patterned arrays of $\mathrm{ZnO}$ nanowires have been obtained but much more control over parameters such as the size of the Au catalyst points and $\mathrm{Zn}$ vapor pressure inside the VPT furnace are required to enable reproducibility and scalability. The second route investigated involved depositing NSL patterned ZnO seeds onto Si directly by PLD. This method was unsuccessful because (a) the low deposition temperatures required to deposit through the PS nanosphere mask mean that the deposited $\mathrm{ZnO}$ seeds were unaligned, leading to randomly oriented VPT growth, and also (b) because $\mathrm{ZnO}$ film growth from the NSL honeycomb patterned $\mathrm{ZnO}$ initial deposit leads to complete substrate coverage and subsequent unpatterned nanowire growth over the entire substrate.

Further work towards the production of large area patterned arrays of nanowires will include replacing PS with silica as the nanosphere material, allowing high temperature PLD depositions though an NSL mask, allowing for the deposition of ordered arrays of high quality textured/aligned $\mathrm{ZnO}$ seeds to catalyze subsequent VPT growth.

\section{Ackowledgements}

The authors gratefully acknowledge funding from SFI under the RFP programme (Grant title: Novel Strategies to enhance the Field Emission Properties of Zinc Oxide Nanowires). 


\section{References}

[1] G.Z Yue, Q. Qiu, Bo Gao, Y. Cheng, J. Zhnag, H. Shimoda, J.P. Lu, O. Zhou, Appl. Phys. Lett. 81 (2002) 355.

[2] J.D. Carey, Philos. Trans. R. Soc. London A 361 (2003), 2891.

[3] Özgür Ü, Alivov Ya I, Liu C, Teke A, Reshchikov M A, Dogan S, Avrutin V, Cho S-J, Morkoç H, J. Appl. Phys. 98 (2005) 041301

[4] Rajendra Kumar R.T, McGlynn E, McLoughlin C, Chakrabarti S, Smith R.C, Carey J.D, Mosnier J.P. and Henry M.O, Nanotechnology 18 (2007) 215704

[5] X. Zhang, D. Liu, L. Zhang, W. Li, M. Gao, W. Ma, Y. Ren, Q. Zeng, Z. Niu, W. Zhou, S. Xie, J. Mater. Chem., 19 (2009) 962-969.

[6] W. Wang, C.J. Summers, Z.L. Wang, Nano Lett., 4(3) (2004) 423-426.

[7] D.F. Liu, Y.J. Xiang, X.C. Wu, Z.X. Zhang, L.F. Liu, L. Song, X. W. Zhao, S. D. Luo, W.J. Ma, J. Shen, W.Y. Zhou, G. Wang, C.Y. Wang, S.S. Xie, Nano Lett., 6(10) (2006) 2375-2378.

[8] H.J. Fan, W. Lee, R. Scholz, A. Dadgar, A. Krost, K. Nielsch, M. Zacharias, Nanotechnology 16 (2005) 913-917.

[9] J. Rybczynski, D. Banerjee, A. Kosiorek, M. Giersig, Z. F. Ren Nano Lett., 4(10) (2004) 2037-2040.

[10] J.-R. Duclère, B. Doggett, M. O. Henry, E. McGlynn, R. T. Rajendra Kumar, J.-P. Mosnier, A. Perrin, and M. Guilloux-Viry, J. Appl. Phys., 101 (2007) 013509.

[11] M. Biswas, E. McGlynn, M. O. Henry, M. McCann, A. Rafferty, J. Appl. Phys. 105, 094306 (2009)

[12] C.L. Haynes, A.D. McFarland, M.T. Smith, J.C. Hulteen, R.P. Van Duyne, J. Phys. Chem. B 106 (2002) 1898-1902.

[13] B.L Zhu, X.H. Sun, X.Z. Zhao, F.H. Su, G.H. Li, X.G. Wu, J. Wu, R. Wu, J. Liu. Vacuum 82 (2008) 495-500. 


\section{List of Figure Captions}

Fig 1: ZnO nanowire array on continuous PLD ZnO buffer layer using NSL patterned Au catalysts (plan view), insets show $45^{0}$ tilted view (upper left, scale bar indicates 2 $\mu \mathrm{m})$ and XRD data (lower right, showing both $\theta-2 \theta$ and rocking curve of $\mathrm{ZnO}(0002)$ peak) illustrating ordering and alignment of nanowire growth.

Fig 2: $\mathrm{ZnO}$ nanowire array on continuous PLD ZnO buffer layer using NSL patterned Au catalysts (plan view), insets show $45^{\circ}$ tilted views at different magnifications (scale bar in inset at upper left is $3 \mu \mathrm{m}$ and at lower right is $2 \mu \mathrm{m}$ ) illustrating ordered growth.

Fig 3: $\mathrm{ZnO}$ film covering substrate after VPT growth on a NSL patterned $\mathrm{ZnO}$ buffer layer in area with no observed VPT nanowire growth. Inset shows the honeycomb $\mathrm{ZnO}$ patterned buffer layer prior to growth. 


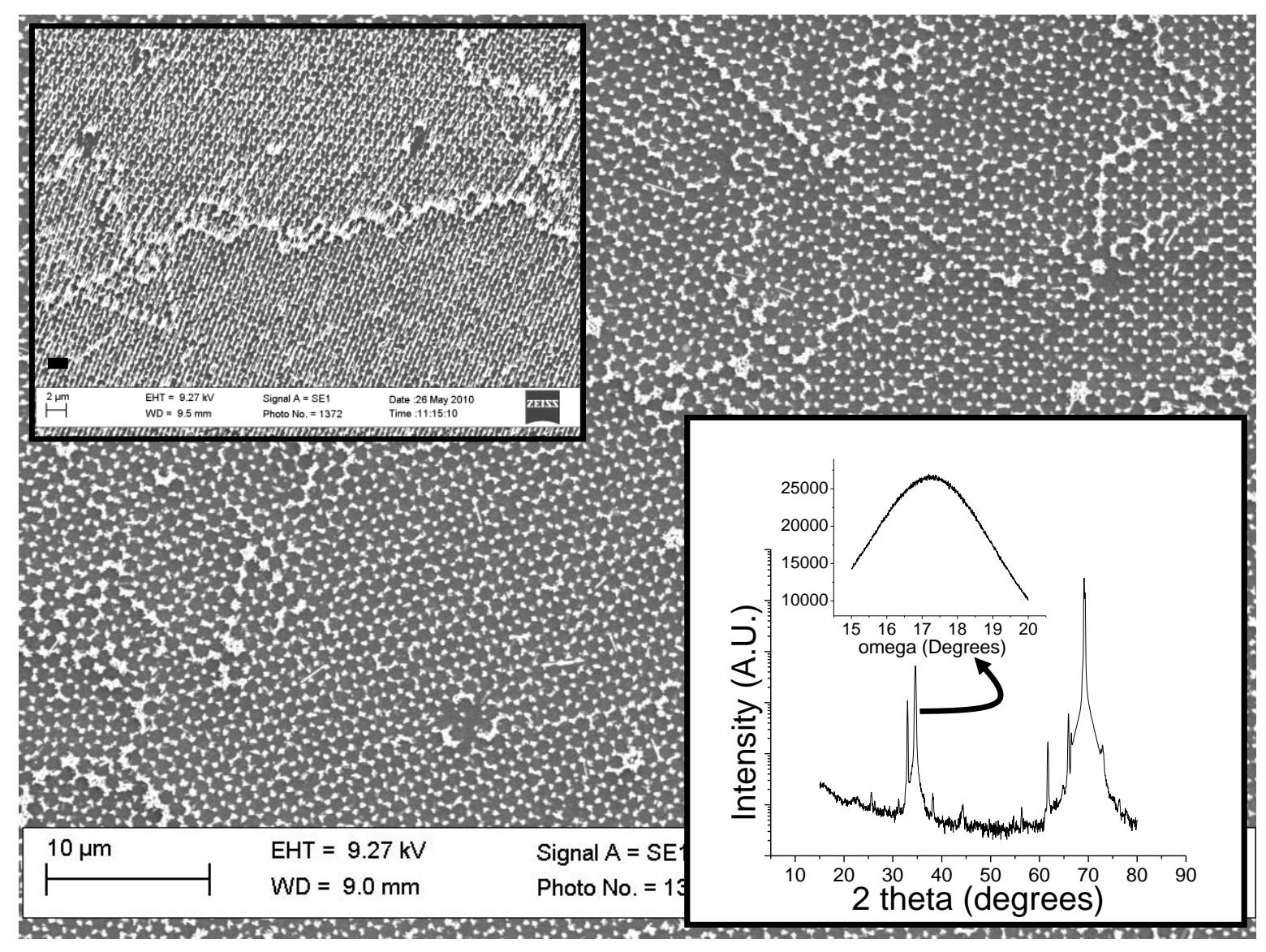

Figure 1. 


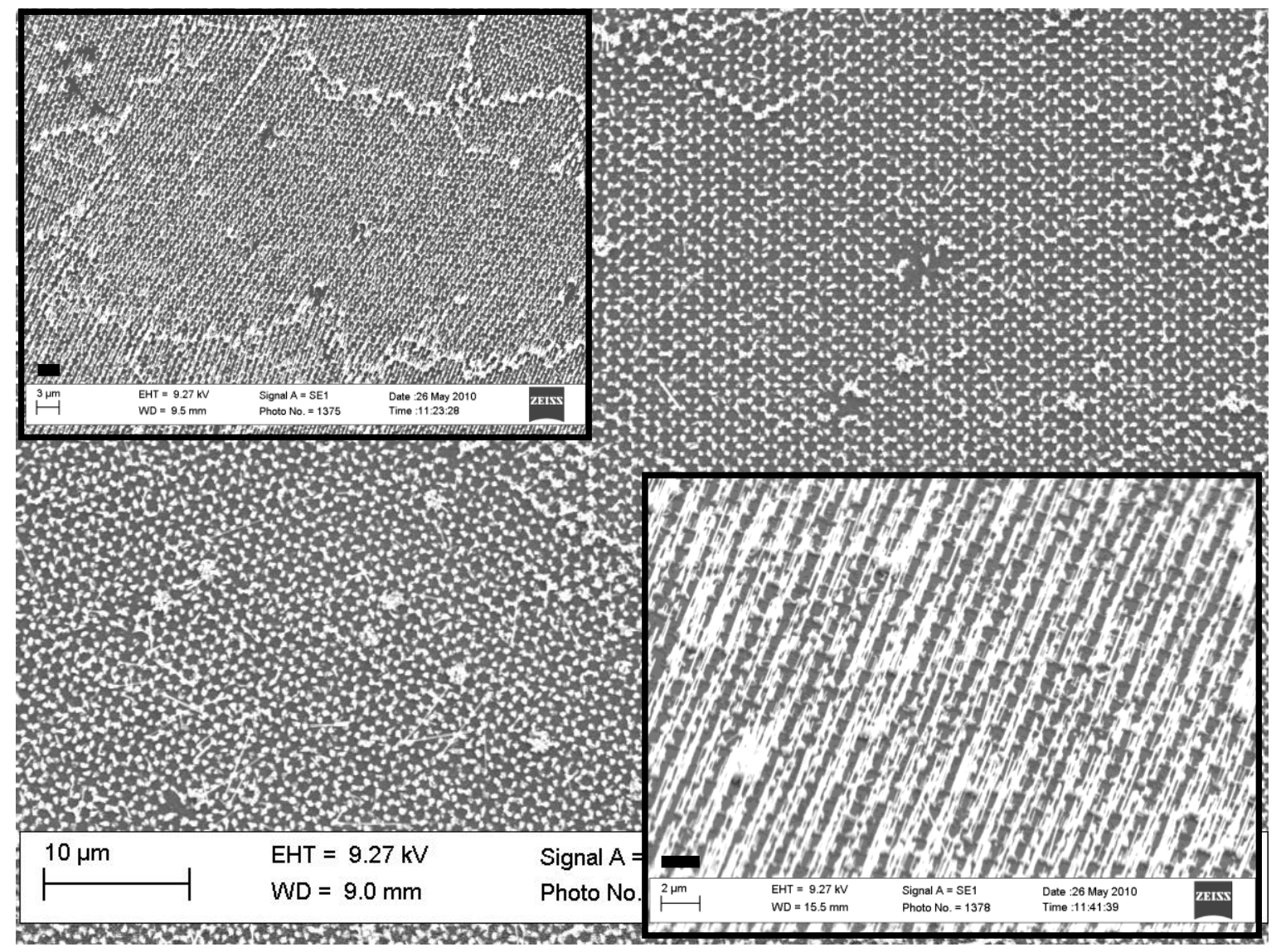

Figure 2. 


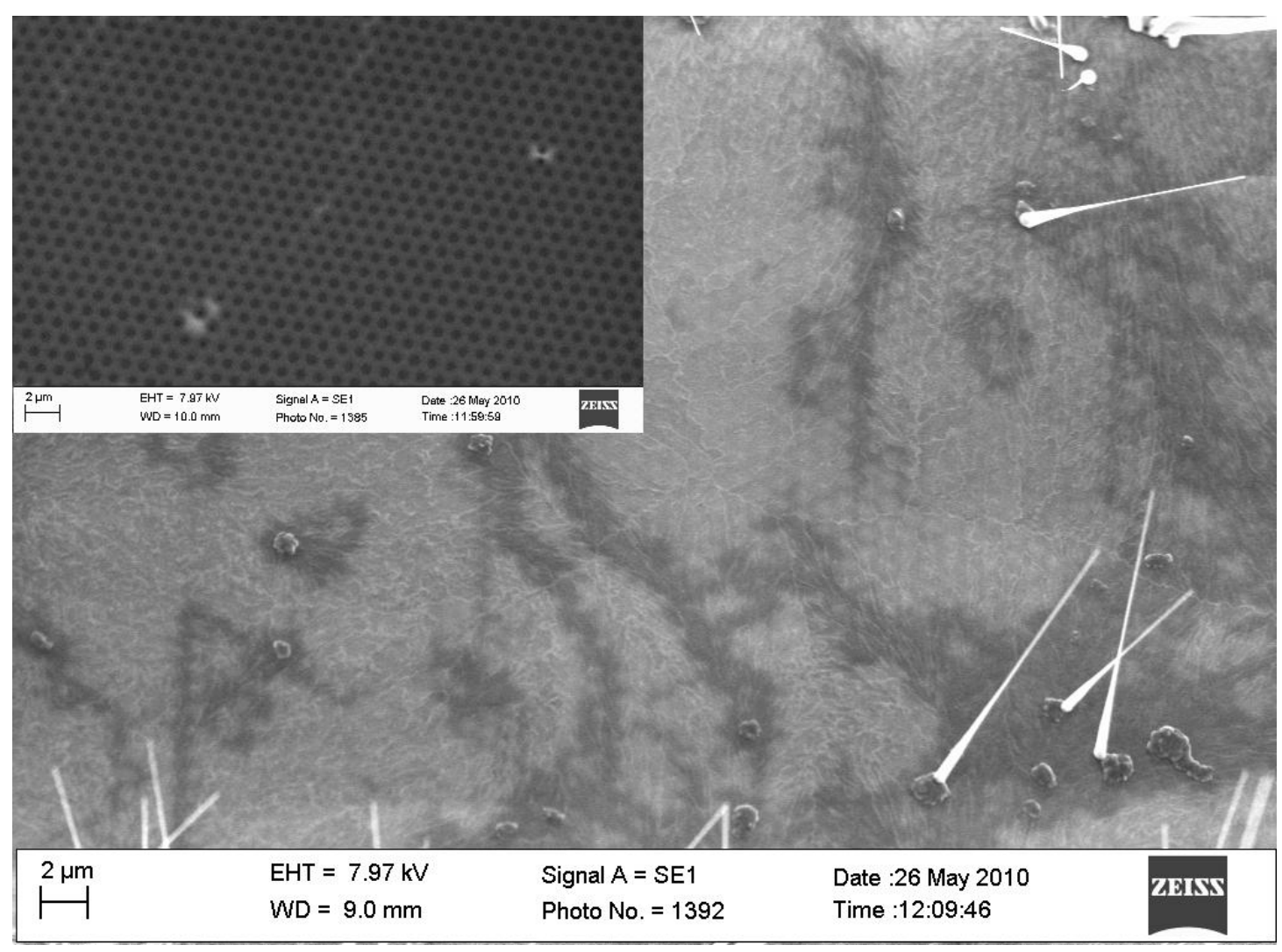

Figure 3 